https://helda.helsinki.fi

\title{
Inter-limb coupling of proximal and distal hand actions
}

\author{
Vainio, Lari
}

2018-01

Vainio , L \& Tiainen, M 2018 , ' Inter-limb coupling of proximal and distal hand actions ' ,

Experimental Brain Research , vol. 236 , no. 1, pp. 153-160 . https://doi.org/10.1007/s00221-017-5114-1

http://hdl.handle.net/10138/311335

https://doi.org/10.1007/s00221-017-5114-1

acceptedVersion

Downloaded from Helda, University of Helsinki institutional repository.

This is an electronic reprint of the original article.

This reprint may differ from the original in pagination and typographic detail.

Please cite the original version. 


\title{
Inter-limb coupling of proximal and distal hand actions
}

\author{
Vainio, L., \& Tiainen, M.
}

Lari Vainio - the corresponding author; Email: lari.vainio@helsinki.fi; Tel: +358-2-94129392

Mikko Tiainen (mikko.o.tiainen@helsinki.fi)

* Department of Psychology and Logopedics, Faculty of Medicine, University of Helsinki, Haartmaninkatu 8, 00014, Finland 


\section{Abstract}

Past studies have revealed connections in directional programming between hands. The present study investigated whether there could also be interaction in programming proximal and distal components of a prehensile hand action. In Experiment 1, the participants performed simultaneously either a push or pull response with the left hand and the grip closure or opening with the right hand. In Experiment 2, the push and pull responses of the left hand were performed together with the precision or power grip responses of the right hand. The participants showed preference, measured in reaction times, to couple the push response with the grip opening and the precision grip, whereas the pull response was associated with the grip closure and the power grip. The study shows for the first time a systematic interaction in proximal and distal prehensile components between two hands. We propose that these effects reflect inter- and intra-limb connections between the representations that prepare the arm extension for the outward reaching, the finger extension for the grip opening, and the motor processes that prepare the precision grip. Conversely, there appears to be connections between the representations that prepare the arm flexion for the inward directed hand movements, the flexion of the thumb and the fingers for the grip closure, and flexion of four fingers for the power grip.

Keywords: action planning, grasping, reaching, inter-limb connections, behavioural study, reaction time 


\section{Introduction}

The present study investigates whether there exists a systematic coupling between proximal hand actions of the left hand and distal hand actions of the right hand. Regarding inter-limb coupling, previous research has revealed connections in directional programming between hands. There appears to be an interaction between homologous muscle groups of two hands; it is easier to perform symmetrical movements of two hands utilizing homologous muscle groups than asymmetrical movements that utilize non-homologous muscle groups (e.g., Swinnen et al. 1997; Serrien et al. 1999). The phenomena of mirror movements and contralateral motor irradiation have shown that corresponding effects can be also observed in increased activity of homologous muscles of the resting hand during voluntary actions of the opposite hand (Cernacek 1961; Armatas et al. 1994).

In general, regarding intra-limb action programming, each neuron in the motor system participates in several hand actions (Schieber and Hibbard 1993). For instance, individual neurons in the motor system do not encode movement direction in an absolute, one-to-one manner, but rather the direction of movement is generated by the coopertation of numerous cells with overlapping tuning curves (Georgipoulos et al. 1982). Furthermore, research has revealed an overlap in activation of contralateral motor representations of distal (thumb, fingers and wrist) and proximal (elbow and shoulder) forelimb movements (e.g., Stark et al. 2007). In line with this evidence, although reaching and grasping are mostly interdependent and programmed in parallel (Jeannerod 1984; Vesia et al. 2010; Vesia et al. 2013) there also appears to be interaction -at least to some extent- in programming reach (i.e., proximal) and grasp (i.e., distal) components of a prehensile action (e.g., Dum and Strick 2005; Raos et al. 2004; Stark et al. 2007; Haggard and Wing 1995).

The grasp component of a prehensile action is typically accomplished by the precision or power-type of grip (Napier 1956). Moreover, both grip types include grip opening and closure phases (Jeannerod 1984). In addition, it could be assumed that some characteristics of reach-tograsp actions are programmed in tighter interaction than some other characteristics. For example, given that outward directed reach-to-grasp is commonly coupled with grip opening, the maximal finger opening occurring within $60-70 \%$ of the duration of the reach (Jeannerod 1984), it could be 
assumed that representations that are commanding forward rather than backward hand movements are tighter integrated with the motor processes that command grip opening.

Intermanual interactions have been previously studied using variety of behavioural methods such as a periodic-movement paradigm (e.g., Swinnen et al. 2001) and a reaction-time paradigm (Diedrichsen et al. 2001). The present study uses the bimanual choice reaction time (RT) task (i.e., the dual-action task) to investigate, for the first time, whether there could be some systematic preference to couple different types of proximal hand movements related to prehensile actions (i.e., reach forwards vs. reach backwards) with the specific grip phase (opening vs. closure: Experiment 1) and/or the specific grip type (precision vs. power: Experiment 2). In general, the bimanual choice RT tasks have been commonly used to investigate the organization of bimanual coordination patterns in the human motor system. For example, an RT advantage has been observed for responses of homologous fingers compared to non-homologous fingers (Wakelin 1976; Heuer 1986). In general, these behavioural studies have been proposed to reveal how mechanisms of separate hands are coupled in action planning (Rosenbaum 1980). The basic principle behind this view is that when the task requires choosing between two alternative responses for both hands according to a single stimulus, the action plans of two alternative responses are prepared in parallel for both hands prior to onset of the stimulus. When then the stimulus is presented and the choice task must be carried out simultaneously for the responses of both hands, there occurs competition between alternative response options. Consequently, the time to select the required motor program for one action is affected by the response selection processes related to the other action. The more the two responses required in each trial share motor characteristics, the less there is interference between selection processes related to two actions, and consequently the faster both actions are executed.

We predict that some characteristics of reach-to-grasp actions such as the arm and finger extension are programmed in tighter interaction than some other characteristics such as the arm extension and the finger flexion. Moreover, we assume that similarly to joint directional programming between hands (Swinnen et al. 1997; Serrien et al. 1999; Armatas et al. 1994), these common prehensile characteristics could be also programmed (to some extent) jointly for both hands. 
As such, according to the view presented above, we hypothesize that if there is partial overlap between processes that plan, for example, forward hand movements and grip opening, dual-action responses should be executed relatively rapidly when one hand is performing grip opening rather than grip closure while the other hand is performing the push movement.

In Experiment1, the participants performed either the push or pull responses with their left hand using a joystick and simultaneously performed either the grip closure or opening with their right hand. Planning the push and pull actions with a joystick can be assumed to utilize the same directional codes as the forward and backward reach actions, respectively. In addition, it can be assumed that execution of the push and pull actions employ the same proximal extension-flexion motor representations as the forward and backward reach actions, respectively. Given that outward directed reach-to-grasp is commonly coupled with grip opening (Jeannerod 1984), we assumed that the participants might prefer to couple the push action of the left hand with the grip opening movement of the right hand. In contrast, the inward directed hand movements are relatively more often associated with moving the grasped object toward the body with fingers wrapped around the object (e.g., bringing an apple to the mouth). Consequently, we presumed that the participants might prefer to couple pull actions with the grip closure in this task.

In addition to grip opening and closure, grasp formation consists of selection of the grip type; that is, preparing to grasp an object using either the precision or power grip. Consequently, if Experiment 1 explores how the proximal prehensile component (i.e., reaching inward vs. outward) is associated with the grip opening and closure processes, Experiment 2 explores how the proximal prehensile component is associated with performing the precision and power grip responses. In Experiment 2, participants performed either the push or pull responses with their left hand and simultaneously performed either the precision grip or power grip with their right hand. Due to the explorative nature of Experiment 2, we did not have specific hypotheses about how push and pull responses would be interacting with the precision and power grip. The precision grip, most commonly used for reaching to grasp small objects, is accomplished by pressing an object between the tip of the index finger and the thumb. In contrast, the power grip is typically accomplished by flexing four fingers toward the palm base so that the thumb does not have an active role in the grip (see, for 
example, Stark et al. 2007). The power grip is mostly implemented by the flexor digitorum profundus (Kilbreath and Gandevia 1994; Reilly and Schieber 2001), which is responsible for flexing index, middle, ring and little finger toward the palm base. Entirely different flexor, namely the flexor pollicis longus, is responsible for flexing the thumb in the precision grip (Kilbreath and Gandevia 1994). Thus, it is possible that the grips would be differently related to the push and pull gestures. As an example, it would be possible that the motor processes that prepare the arm flexion of the left hand are more tightly integrated with the motor processes that prepare the flexion of the fingers for the power grip than the flexion of the thumb for the precision grip. This would be observed in coupling between the backward directed hand movement of the left hand and the power grip of the right hand.

\section{Methods}

\section{Participants}

In Experiment 1, nineteen naïve volunteers participated in the study (19-36 years of age; mean age $=24.4$ years; 2 males). In Experiment 2, eighteen naïve volunteers participated in the study (22-31 years of age; mean age $=25.5$ years; 5 males). These sample sizes were chosen based on our previous knowledge of effect sizes in these kinds of experiments (Vainio et al. 2015; Tiainen et al. 2017), and we estimated that with these sample sizes, we should reach Power around 0.9. All participants were psychology students of University of Helsinki. The gender was not considered as a criteria for selecting participants because the preliminary analyses of our previous studies, in which the grip and joystick responses were similarly performed in a dual-action tasks (Vainio et al. 2015; Tiainen et al. 2017) indicated that gender does not seem to play a significant role in these kinds of behavioural effects. The most of the participants were female because approximately $80 \%$ of the psychology students are female in University of Helsinki. All participants were right-handers and had normal or corrected-to-normal vision. 


\section{Compliance with Ethical Standards}

The study was approved by the Ethical Committee of the Institute of Behavioural Sciences at the University of Helsinki and has therefore been performed in accordance with the ethical standards laid down in the 1964 Declaration of Helsinki. Written informed consent was obtained from all participants.

\section{Apparatus, stimuli and procedure}

Each participant sat in a dimly lit room with his or her head $70 \mathrm{~cm}$ in front of a 19 -in. CRT monitor (screen refresh rate: $85 \mathrm{~Hz}$; screen resolution: $1280 \times 1024)$. The target stimuli consisted of two horizontally oriented lines (length: $2^{\circ}$ ) from which one line was clearly thicker than the other line (thin: $0.2^{\circ}$; thick: $0.4^{\circ}$ ). The thin line was either below or above the thick line (Fig. 1 ). In addition, both lines were presented either in green or blue color. All stimuli were displayed in the center of the monitor on a white background.

In the trial structure (see Fig. 1), a fixation cross was presented for $400 \mathrm{~ms}$ at the beginning of each trial. Then, a blank screen was displayed for $400 \mathrm{~ms}$. After that the target stimulus was presented 2000 ms or until a response was executed with both hands. Finally, a blank screen was displayed for 500 ms. Both experiments began with practice trials. Each participant was given as much practice as it took to perform the task fluently (i.e., fast and simultaneous left and right hand responses with a small number of errors).

In both experiments, the participants' left-hand task was to pull or push the joystick until the end of the full motion range of the joystick ( $4.5 \mathrm{~cm}$ forward or backward) as fast and accurately as possible according to the spatial arrangement of the double line stimuli (see Fig. 1). Half of the participants responded according to the relative location of the thin line (e.g., move the hand forwards if the thin line is above the thick line and backward if it is below the thick line) while half of the participants responded according to the relative location of the thick line. The joystick was always returned to the central starting position after the response. The joystick was attached steadily onto 
the table and located between the participant and the monitor, $30 \mathrm{~cm}$ away from the monitor. It was positioned horizontally $10 \mathrm{~cm}$ to the left of the center of the monitor.

In Experiment 1, the right hand responses were performed with the scissors (see Fig. 1) by opening or closing them at the same time with the left hand response. The scissors were equipped with two microswitches from which one registered the opening of the scissors and the other registered the closing of them. The scissors were opened and closed by the handles using index and middle finger in the one handle and the thumb in the other handle. The grip opening versus closure was performed according to the color of the stimulus (e.g., blue for the opening and green for the closing). The microswitches of the scissors were marked with color tapes that corresponded to the required color-movement mapping arrangements. After the opening/closure response was executed, the participants were required to return the scissors back to the half open position. Two scissor blades were attached by a screw (see Fig. 1), and twisting this screw enabled manipulating stiffness of the closing movement. When the screw was loose, there was no any tactually observable resistance in the closing movement. The extent that the screw was tightened defined the extent of the resistance of the closing movement as well as at which point of the closing movement, from fully open to fully close, the resistance began. Stiffness of the scissors was adjusted so that they provided tactually observable resistance at the point from which there was approximately equal motion range $(2 \mathrm{~cm})$ toward the maximal open and the maximal close position of the scissors.

In Experiment 2, the right-hand responses were performed with the precision $(1 \times 1 \times$ $0.7 \mathrm{~cm}$ ) and power (10 cm long, $3 \mathrm{~cm}$ diameter) grip devices (see Fig. 1) by pressing one of the devices at the same time with the left hand response. The devices were equipped with an inlaid microswitch. As the switches were depressed in each device, there was noticeable tactile feedback. The participants were required to perform the precision or power grip response according to the color of the stimulus (e.g., blue for the precision grip and green for the power grip). The grip devices were marked with corresponding color tapes that corresponded to the required color-grip mapping arrangements. The grip task and the devices were adapted from the study reported by Tucker and Ellis (2001; Vainio et al. 2008). Because both of the grip devices were held in the right hand, the contribution of the index finger -typically also involved in the power grip- was missing in the power 
grip task, which had to be executed using the little, ring and middle finger. Otherwise these grip actions were performed exactly in the way they are normally performed (see the descriptions in the Introduction).

The color-movement (Experiment 1) and color-grip (Experiment 2) mapping was balanced so that approximately half of the participants performed closing/precision action for the blue color, while rest of the participants performed closing/precision action for the green color, in both of the joystick response arrangements that determined push-pull responses according to the relative spatial location of the thin or thick line. In addition, in both experiments, the right hand was resting on the arm of an armchair while holding the devices.

Reaction times (RTs) of the joystick movements were measured from the onset of the target stimulus to the point of the joystick movement in which the joystick reached $100 \%$ from the motion range of the joystick to the forward or backward direction. The response was registered as an 'error' if joystick movement exceeded $100 \%$ of the motion range but was moved into the wrong direction. In addition, the response was registered as 'no-response' if the joystick movement did not reach $100 \%$ of the motion range. Regarding the close/open and precision/power responses, RTs were measured from the onset of the target stimulus to the point of the response in which the movement resulted in the pushing one of the microswitches of the device.

\section{Statistical analysis}

Experiment 1 consisted of 140 trials [35 (repetition) $\times 2$ (left-hand: push/pull) $\times 2$ (right-hand: close/open)]. Statistical significance of reaction time values was tested by using a random intercept model (Linear Mixed Models; see Barr et al. 2013 for a justification of using this statistical method with the current data) that treated Joystick (push or pull), Scissors (open or close) and Mapping (between subjects) [MP1 (green/open or MP2 (green/close)] as fixed factors and Subject as a random intercept. Post hoc comparisons were carried out by using the Bonferroni correction. Regarding the joystick reaction times, the post hoc comparisons tested whether the joystick responses were modulated by the scissor responses; That is, whether one type of joystick response 
(e.g., push) was performed significantly faster/slower together with one type of scissor response (e.g., open) compared to other type of scissor response (e.g., close). Regarding the scissor reaction times, the post hoc comparisons tested whether the scissor responses were modulated by the joystick responses; That is, whether one type of scissor response (e.g., close) was performed significantly faster/slower together with one type of joystick response (e.g., pull) compared to other type of joystick response (e.g., push).

Experiment 2 consisted of 160 trials [40 (repetition) $\times 2$ (left-hand: push/pull) $\times 2$ (righthand: precision/power)]. Statistical significance of reaction time values was tested by using a random intercept model (Linear Mixed Models) that treated Joystick (push or pull), Grip (precision or power) and Mapping (between subjects) [MP1 (green/precision or MP2 (green/power)] as fixed factors and Subject as a random intercept. Post hoc comparisons were carried out by using the Bonferroni correction. Regarding the joystick reaction times, the post hoc comparisons tested whether the joystick responses were modulated by the grip responses; That is, whether one type of joystick response (e.g., push) was performed significantly faster/slower together with one type of grip response (e.g., precision) compared to other type of grip response (e.g., power). Regarding the grip reaction times, the post hoc comparisons tested whether the grip responses were modulated by the joystick responses; That is, whether one type of grip response (e.g., power) was performed significantly faster/slower together with one type of joystick response (e.g., pull) compared to other type of joystick response (e.g., push).

---Figure 1 about here---

\section{Results}

\section{Experiment 1}

$6.4 \%$ of the raw data containing errors were discarded from the analysis of reaction times of joystick and scissor responses. The discarded error data consisted of those responses that were incorrectly 
executed with the left hand $(2.2 \%)$, right hand $(3.8 \%)$ or with both hands $(0.4 \%)$. In addition, regarding the left-hand (joystick) responses, $4.5 \%$ of trials in which the RTs were more than two standard deviations from a participant's overall mean were discarded from the analysis. Regarding the right-hand (scissor) responses, $4.4 \%$ of trials in which the RTs were more than two standard deviations from a participant's overall mean were additionally discarded from the analysis.

The errors were not analyzed for the joystick or scissor responses because the participants produced so few errors. The analysis of Joystick responses revealed significant main effects of Joystick $[F(1,2359)=6.09, p=.014]$ and Scissor $[F(1,2359)=5.79, p=.016]$. The backward responses were made faster $(M=930 \mathrm{~ms}, 95 \% \mathrm{Cl}[842,1018])$ than the forward responses $(M=953 \mathrm{~ms}, 95 \% \mathrm{Cl}[865,1040])$. In addition, the close responses were made faster $(M$ $=930 \mathrm{~ms}, 95 \% \mathrm{Cl}[843,1018])$ than the open responses $(M=952 \mathrm{~ms}, 95 \% \mathrm{Cl}[865,1040])$. Joystick x Scissor interaction was also obtained $[F(1,2359)=52.81, p<.001]$. This interaction is presented in Fig. 2. The pairwise comparisons test showed that the push responses were performed faster when the scissors were opened $(M=930 \mathrm{~ms}, 95 \% \mathrm{Cl}[842,1019])$ rather than closed $(M=975 \mathrm{~ms}$, $95 \% \mathrm{Cl}[886,1063])(p=.001)$. In contrast, the pull responses were performed faster when the scissors were closed $(M=886 \mathrm{~ms}, 95 \% \mathrm{Cl}[798,975])$ rather than opened $(M=974 \mathrm{~ms}, 95 \% \mathrm{Cl}$ $[886,1063])(p<.001)$. The interaction between Joystick, Scissor and Mapping was not significant $[F(1,2359)=0.88, p=.348]$ suggesting that Mapping (i.e., which color cued opening/closing responses) did not interfere with the interaction between the joystick and scissor responses.

The analysis of Scissor responses revealed significant main effects of Scissor $[F(1,2357)=8.29, p=.004]$. The close responses were made faster $(M=907 \mathrm{~ms}, 95 \% \mathrm{Cl}[812$, 1002]) than the open responses $(M=934 \mathrm{~ms}, 95 \% \mathrm{Cl}[839,1029])$. Joystick $\mathrm{x}$ Scissor interaction was also obtained $[F(1,2357)=50.33, p<.001]$. This interaction is presented in Fig. 2 . The pairwise comparisons test showed that the open responses were performed faster when the joystick was pushed $(M=906 \mathrm{~ms}, 95 \% \mathrm{Cl}[810,1001])$ rather than pulled $(M=962 \mathrm{~ms}, 95 \% \mathrm{Cl}[866,1058])(p$ $<.001)$. In contrast, the close responses were performed faster when the joystick was pulled $(M=$ $869 \mathrm{~ms}, 95 \% \mathrm{Cl}[773,965])$ rather than pushed $(M=944 \mathrm{~ms}, 95 \% \mathrm{Cl}[849,1040])(p<.001)$. The interaction between Joystick, Scissor and Mapping was not significant $[F(1,2357)=1.64, p=.201]$. 
We also analyzed the reaction time difference between the left and right hand responses (joystick RTs - scissor RTs). The reaction times of the bimanual responses were closely synchronized (mean left hand lead $=20 \mathrm{~ms}$, standard error $=8.5 \mathrm{~ms}$ ). In the analysis of the reaction time difference, in which the Joystick and Scissors were treated as fixed factors and Subject as a random intercept, did not reveal interaction between the joystick and scissor responses $[F(1,2344)$ $=0.09, p=.766]$ suggesting that the synchrony between the joystick and scissor responses is similar in conditions that are hypothesized to be congruent (i.e., push-open and pull-close) and incongruent (i.e., push-close and pull-open).

---Figure 2 about here---

\section{Experiment 2}

$3.3 \%$ of the raw data containing errors were discarded from the analysis of reaction times of joystick and grip responses. The discarded error data consisted of those responses that were incorrectly executed with the left hand $(1.2 \%)$, right hand $(1.8 \%)$ or with both hands $(0.3 \%)$. In addition, regarding the left-hand (joystick) responses, $4.6 \%$ of trials in which the RTs were more than two standard deviations from a participant's overall mean were discarded from the analysis. Regarding the right-hand (grip) responses, $4.4 \%$ of trials in which the RTs were more than two standard deviations from a participant's overall mean were additionally discarded from the analysis.

The errors were not analyzed for the joystick or scissor responses because the participants produced so few errors. The analysis of Joystick responses revealed significant main effects of Joystick $[F(1,2635)=38.25, p<.001]$ and Grip $[F(1,2635)=4.76, p=.029]$. Similarly, to Experiment 1, the backward responses were made faster $(M=759 \mathrm{~ms}, 95 \% \mathrm{Cl}[693,824])$ than the forward responses $(M=788 \mathrm{~ms}, 95 \% \mathrm{Cl}[723,854])$. In addition, the precision responses were made faster $(M=768 \mathrm{~ms}, 95 \% \mathrm{Cl}[703,834])$ than the power responses $(M=779 \mathrm{~ms}, 95 \% \mathrm{Cl}[712,844])$. Joystick x Grip interaction was also obtained $[F(1,2635)=60.10, p<.001]$. This interaction is presented in Fig. 3. The pairwise comparisons test showed that the push responses of the left hand were performed faster when the right hand was making the precision responses $(M=764 \mathrm{~ms}, 95 \%$ 
$\mathrm{Cl}[698,830])$ rather than the power responses $(M=812 \mathrm{~ms}, 95 \% \mathrm{Cl}[746,878])(p<.001)$. In contrast, the pull responses were performed faster when the right hand was making the power responses $(M=745 \mathrm{~ms}, 95 \% \mathrm{Cl}[679,811])$ rather than the precision responses $(M=771 \mathrm{~ms}, 95 \%$ $\mathrm{CI}[706,838])(p<.001)$. The interaction between Joystick, Grip and Mapping was not significant $[F(1,2635)=1.70, p=.192]$.

The analysis of Grip responses revealed significant main effects of Grip $[F(1,2640)=$ 4.26, $p=.039]$. The precision responses were made faster $(M=764 \mathrm{~ms}, 95 \% \mathrm{Cl}[682,845])$ than the power responses $(M=774 \mathrm{~ms}, 95 \% \mathrm{Cl}[692,855])$. Joystick x Grip interaction was also obtained $[F(1,2640)=58.72, p<.001]$. This interaction is presented in Fig. 3. The pairwise comparisons test showed that the precision responses were performed faster when the joystick was pushed $(M=749$ $\mathrm{ms}, 95 \% \mathrm{Cl}[667,831])$ rather than pulled $(M=779 \mathrm{~ms}, 95 \% \mathrm{Cl}[697,860])(p<.001)$. In contrast, the power responses were performed faster when the joystick was pulled ( $M=751 \mathrm{~ms}, 95 \% \mathrm{Cl}[669$, 833]) rather than pushed $(M=797 \mathrm{~ms}, 95 \% \mathrm{Cl}[715,878])(p<.001)$. The interaction between Joystick, Grip and Mapping was not significant $[F(1,2640)=0.01, p=.926]$.

We also analyzed the reaction time difference between the left and right hand responses (joystick $\mathrm{RTs}$ - grip RTs). The reaction times of the bimanual responses were closely synchronized (mean left hand lead $=6 \mathrm{~ms}$, standard error $=18.5 \mathrm{~ms}$ ). The analysis was similar as in Experiment 1, with Joystick and Grip as the fixed factors, and did not reveal interaction between the joystick and grip responses $[F(1,2610)=0.12, p=.734]$.

---Figure 3 about here---

\section{Discussion}

The present study demonstrated preference to couple the forward hand movement (i.e., proximal prehensile component) with the grip opening and the precision grip (i.e., distal prehensile components) whereas the backward hand movement was preferably coupled with the grip closure and the power grip. These interactions were observed even though the proximal and distal actions 
were performed with separate hands. This is the first time that the processes involved in planning of the specific proximal and distal prehensile actions are shown to interact in a systematic manner between two hands.

We propose that the push action was connected to the grip opening because both actions are related to an effector extension: in the push action, the arm is extended, whereas in the grip opening, the thumb and the fingers are extended. Similarly, we assume that the pull action was connected to the grip closure because both actions are related to an effector flexion: in the pull action, the arm is flexed, whereas in the grip closure, the thumb and the fingers are flexed. However, it should be noted that the push and pull actions are accomplished by moving the elbow as well as the shoulder. Importantly, in the push action, the elbow is extended whereas the shoulder is flexed (Lacquaniti and Soechting 1982). In contrast, in the pull action, the elbow is flexed whereas the shoulder is extended. Consequently, if the present interaction effect is indeed based on intra- and inter-limb connections between processes that prepare extension-flexion movements of proximal and distal components related to prehensile actions, it has to be exclusively related to elbow-related movement planning instead of shoulder-related movement planning. We should have observed the opposite interaction effect if the effect would operate in relation to motor processes that prepare shoulder extension-flexion movements.

Regarding the interaction between the push-pull and the precision-power responses observed in Experiment 2, similarly to the effect observed in Experiment 1, the interaction can be based on intra- and inter-limb connections between processes that prepare extension-flexion movements of proximal and distal components related to prehensile actions. According to this view, given that the power grip is accomplished by flexing four fingers toward the palm base mostly via the flexor digitorum profundus (Kilbreath and Gandevia 1994; Reilly and Schieber 2001), we propose that the pull response was preferably coupled with the power grip because there are intra- and interlimb connections between motor representations that control elbow flexion and flexion of the four fingers.

In contrast to the power grip, the precision grip is mostly implemented by the flexor pollicis longus, which is responsible for flexing the thumb in this grip (Kilbreath and Gandevia 1994). 
Regarding the fingers that are typically actively flexed in the power grip (i.e., the little, ring and middle finger), the flexion of these fingers has to be inhibited in the precision grip, and in some extreme types of the pincer grip (e.g., when one is, for example, pinch gripping a needle) these fingers might be even extended (McMillan and Carin-Levy 2012). Similarly, in the current paradigm, when the task required executing the precision grip response, the accurate performance required flexor inhibition of the little, ring and middle fingers in order to withhold incorrectly performing the power grip. According to this view, when the participant had to select the precision grip for the response, the selection was executed relatively slowly when the precision grip response was coupled with the pull action because the pull action is associated with active flexion of the fingers, consequently favoring the power grip rather than the precision grip.

An alternative reason why the precision grip was associated with the push response assumes developmental links between outward directed reach action and the precision grip. It has been proposed that learning the precision grasp action precedes the development of hand pointing action (Leung and Rheingold 1981; Murphy and Messer 1977) in which the central component is the arms extension away from the body. According to this view, outward pointing action is adjusted from the precision grasp action by the end of the first year. Consequently, it is possible that there are developmentally-driven connections between processes that prepare forward directed hand movements and the precision grip, which in turn manifest themselves in the present findings.

In previous studies that have used reaction time paradigm in order to explore intermanual interactions, reaction times have been associated with the onset (reaction time onset RT) or the offset (movement time - MT) of, for example, the aiming movements (Diedrichsen et al. 2006; Heuer \& Klein, 2006). It has been previously also suggested that the congruency effects in dual-action tasks exploring intermanual interactions can be based on 1) interferences in assigning the specific stimulus to the correct response and/or 2) movement related conflicts reflecting interactions of movement parameters (e.g., Diedrichsen et al. 2006). In addition, it might be assumed, for example, that RTs are more sensitive to interferences in stimulus-response mapping processes whereas MTs are more sensitive to movement related conflicts. Hence, given that RTs and MTs might reflect different underlying mechanisms in the effects of intermanual interactions, the inability 
to report the congruency effects in relation to RTs as well as MTs can be considered as one of the potential weaknesses of the present study. The response devices of the study did not allow measuring RTs as well as MTs. In Experiments 1 and 2, the left-hand (joystick) responses were measured in relation to the offset of the movement. The right-hand responses of Experiment 1 were similarly measured in relation to offset of the opening/closing movement. In contrast, the right-hand responses of Experiment 2 were measured in relation to squeezing the precision of power grip devices. As such, the grip devices did not allow measurement of any movement times. Consequently, we cannot be certain whether processes related to mapping the specific stimulus to the correct response could also have some role in the effects or whether the effects purely reflect interactions of movement parameters.

In conclusion, the present study shows a systematic interaction in proximal and distal prehensile components between two hands. The forward directed movement of the left hand was associated with the grip opening and the precision grip performed with the right hand. In contrast, the backward directed movement of the left hand was associated with the grip closure and the power grip performed with the right hand. The previous research exploring preferences to couple dualactions simultaneously performed with two hands have mostly focused on directional aspects of the two movements showing, for example, a general preference to produce movements of two hands in the same direction in comparison to different directions (e.g., Swinnen et al. 1997; Serrien et al. 1999). The present study shows that, in addition to these inter-limb connections related to directional coding, the two hands also share motor programming processes in relation to planning proximal and distal prehensile components. Processes involved in planning the proximal prehensile action for one hand have systematic influence on processes involved in planning the distal prehensile action for another hand, and vice versa. In better detail, we propose that the motor system includes relatively tight inter- and intra-limb connections between the representations that prepare the elbow extension for the outward directed reaching, the finger extension for the grip opening, and the mechanisms that operate for preparing the precision grip. In addition, corresponding connections might be operating between the representations that prepare the elbow flexion for the inward directed arm 
movements, the flexion of fingers and the thumb for the grip closure, and the flexion of the four fingers for the power grip.

\section{Acknowledgments}

We are very grateful to Aleksi Rantala for helping us collecting the data. Many thanks to Martti Vainio and Kaisa Tiippana for getting involved in planning these studies. The research leading to these results has received funding from the Academy of Finland under grant agreement numbers 1265610.

\section{Conflict of interest}

The authors declare that they have no conflict of interest. 


\section{References}

Armatas CA, Summers JJ, Bradshaw JL (1994) Mirror movements in normal adult subjects. J Clin Exp Neuropsychol 16:405-413

Barr DJ, Levy R, Scheepers C, Tily HJ (2013) Random effects structure for confirmatory hypothesis testing: Keep it maximal. J Mem Lang 68:255-278

Cernacek J (1961) Contralateral motor irradiation-cerebral dominance: its changes in hemiparesis. Arch Neurol 4:165-172

Diedrichsen J, Hazeltine E, Kennerley S, Ivry RB (2001) Moving to directly cued locations abolishes spatial interference during bimanual actions. Psychol Sci 12:493-498

Diedrichsen J, Grafton S, Albert N, Hazeltine E, Ivry RB (2006) Goal-selection and movement-related conflict during bimanual reaching movements. Cerebral Cortex 16:1729-1738

Dum RP, Strick PL (2005) Frontal lobe inputs to the digit representations of the motor areas on the lateral surface of the hemisphere. J Neurosci 25:1375-1386

Georgopoulos AP, Kalaska JF, Caminiti R, Massey JT (1982) On the relations between the direction of two-dimensional arm movements and cell discharge in primate motor cortex. J Neurosci 2:15271537

Haggard P, Wing A (1995) Coordinated responses following mechanical perturbation of the arm during prehension. Exp Brain Res 102:483-494 
Heuer H (1986) Intermanual interactions during programming of finger movements: transient effects of 'homologous coupling'. Generation and modulation of action patterns 15:87-101

Heuer $\mathrm{H}$, Klein W (2006) The modulation of intermanual interactions during the specification of the directions of bimanual movements. Exp Brain Res 169:162-181

Jeannerod M (1984) The timing of natural prehension movements. J Mot Behav 16:235-254

Kilbreath SL, Gandevia SC (1994) Limited independent flexion of the thumb and fingers in human subjects. J Physiol 479:487

Lacquaniti F, Soechting JF (1982) Coordination of arm and wrist motion during a reaching task. J Neurosci 2:399-408

Leung EH, Rheingold HL (1981) Development of pointing as a social gesture. Dev Psychol 17:215 Murphy CM, Messer D (1977) Mothers, infants, and pointing: a study of a gesture. In: Schaffer HR (ed) Studies in mother-infant interaction. Academic Press, London, pp 325-354

Napier JR (1956) The prehensile movements of the human hand. Bone Joint J 38:902-913

Raos V, Umiltá MA, Gallese V, Fogassi L (2004) Functional properties of grasping-related neurons in the dorsal premotor area F2 of the macaque monkey. J Neurophysiol 92:1990-2002

Reilly KT, Schieber MH (2001) Electromyographic (EMG) activity of functional subdivisions in human multitendoned finger muscles. In Society of Neuroscience Abstracts (Vol. 27, No. 938.3) 
Rosenbaum DA (1980) Human movement initiation: specification of arm, direction, and extent. J Exp Psychol Gen 109:444

Schieber MH, Hibbard LS (1993) How somatotopic is the motor cortex hand area?. SCIENCE-NEW YORK THEN WASHINGTON-, 489-489

Serrien DJ, Bogaerts H, Suy E, Swinnen SP (1999) The identification of coordination constraints across planes of motion. Exp Brain Res 128:250-255

Stark E, Asher I, Abeles M (2007) Encoding of reach and grasp by single neurons in premotor cortex is independent of recording site. J Neurophysiol 97:3351-3364

Swinnen SP, Jardin K, Meulenbroek R, Dounskaia N, Hofkens-Van Den Brandt M (1997) Egocentric and allocentric constraints in the expression of patterns of interlimb coordination. $\mathrm{J}$ Cogn Neurosci 9:348-377

Swinnen SP, Dounskaia N, Levin O, Duysens J (2001) Constraints during bimanual coordination: the role of direction in relation to amplitude and force requirements. Behav Brain Res 123:201-218

Tiainen M, Tiippana K, Vainio M, Komeilipoor N, Vainio L (2017) Interaction in planning vocalizations and grasping. Q J Exp Psychol 70: 1590-1602

Tucker M, Ellis R (2001) The potentiation of grasp types during visual object categorization. Vis Cogn 8:769-800

Vainio L, Symes E, Ellis R, Tucker M, Ottoboni G (2008) On the relations between action planning, object identification, and motor representations of observed actions and objects. Cogn 108:444-465 
Vainio L, Tiainen M, Tiippana K, Komeilipoor N, Vainio M (2015) Interaction in planning movement direction for articulatory gestures and manual actions. Exp Brain Res 233:2951-2959

Vesia M, Prime SL, Yan X, Sergio LE, Crawford JD (2010) Specificity of human parietal saccade and reach regions during transcranial magnetic stimulation. J Neurosci 30: 13053-13065

Vesia M, Bolton DA, Mochizuki G, Staines WR (2013) Human parietal and primary motor cortical interactions are selectively modulated during the transport and grip formation of goal-directed hand actions. Neuropsychologia 51:410-417

Wakelin DR (1976) The role of the response in psychological refractoriness. Acta psychol 40:163175 


\section{Figure captions}

Fig. 1 Graphical presentation of the trial structure of Experiments 1 and 2 (see text for details). In both experiments, the left-hand responses were performed with the joystick by moving it forwards or backwards. The right hand responses were performed with the scissors (Experiment 1) by the finger opening or closure gesture, or with the grip device by the precision or power grip (Experiment 2). The scissors and the grip device were equipped with microswitches (their location is marked with the dotted circles in the picture) that registered the responses.

Fig. 2 The mean vocal reaction times for Experiment 1 as a function of the joystick (i.e., the left-hand) and the scissor (i.e., the right-hand) responses. Error bars depict the standard error of the mean. Asterisks indicate statistically significant differences $\left({ }^{* * *}<.001 ;{ }^{* *} \mathrm{p}<.01 ;{ }^{*} \mathrm{p}<.05\right)$.

Fig. 3 The mean vocal reaction times for Experiment 2 as a function of the joystick (i.e., the left-hand) and the grip (i.e., the right-hand) responses. Error bars depict the standard error of the mean. Asterisks indicate statistically significant differences $\left({ }^{* * *}<.001 ;{ }^{* *} \mathrm{p}<.01 ;{ }^{*} \mathrm{p}<.05\right)$. 
Figure 1

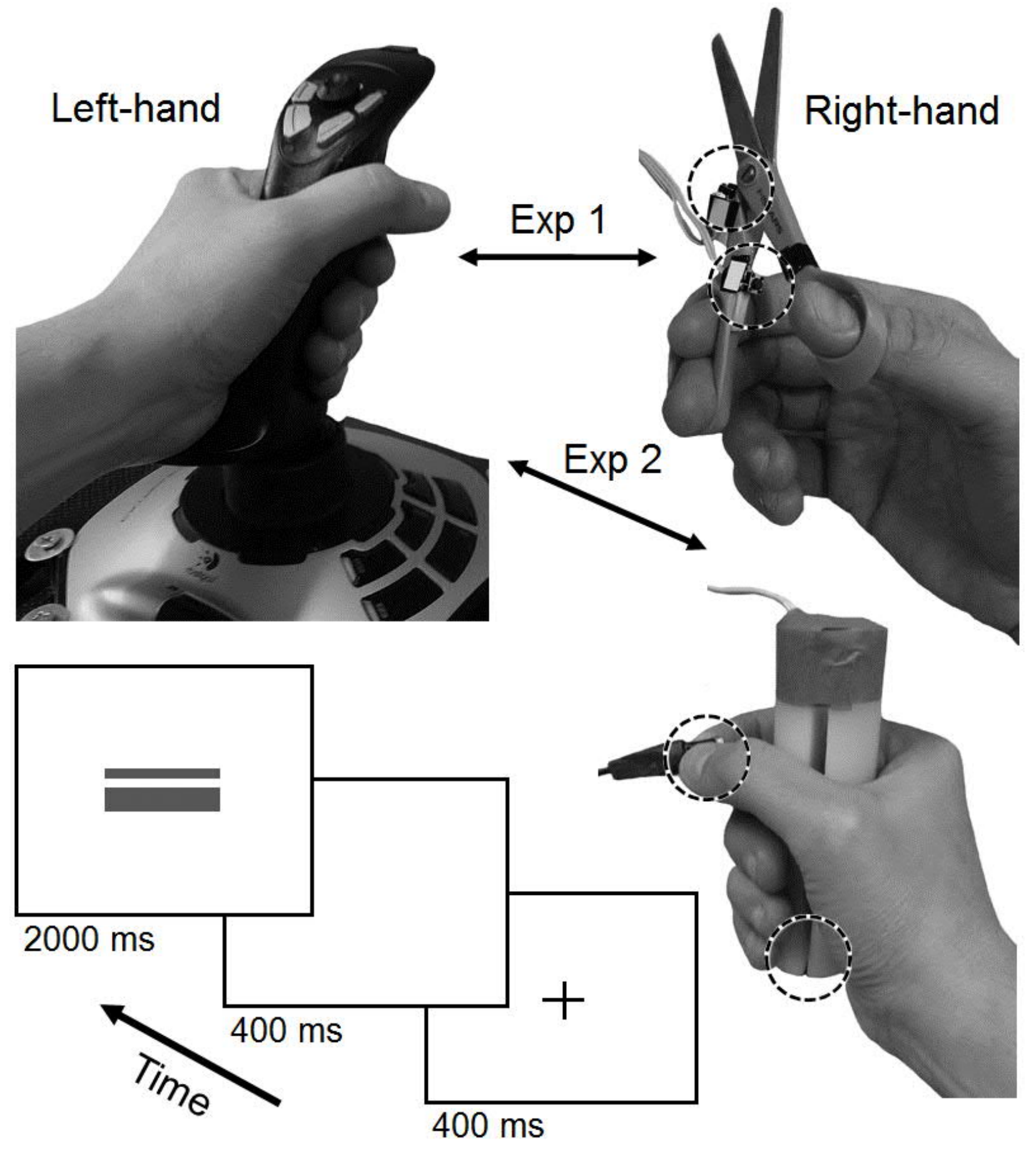


Figure 2

Joystick (Left-hand) Scissors (Right-hand)

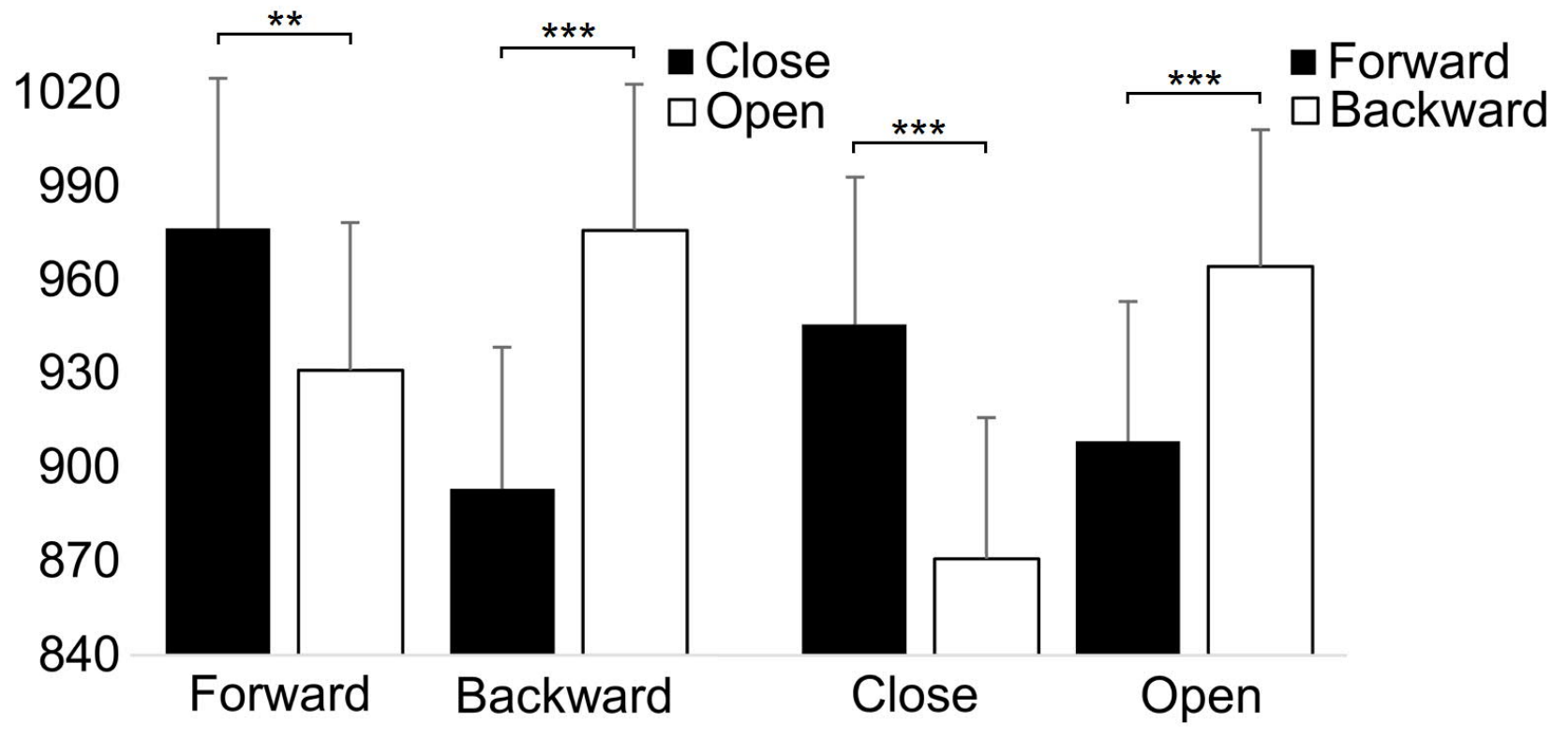

Figure 3

Joystick (Left-hand) Grip device (Right-hand)

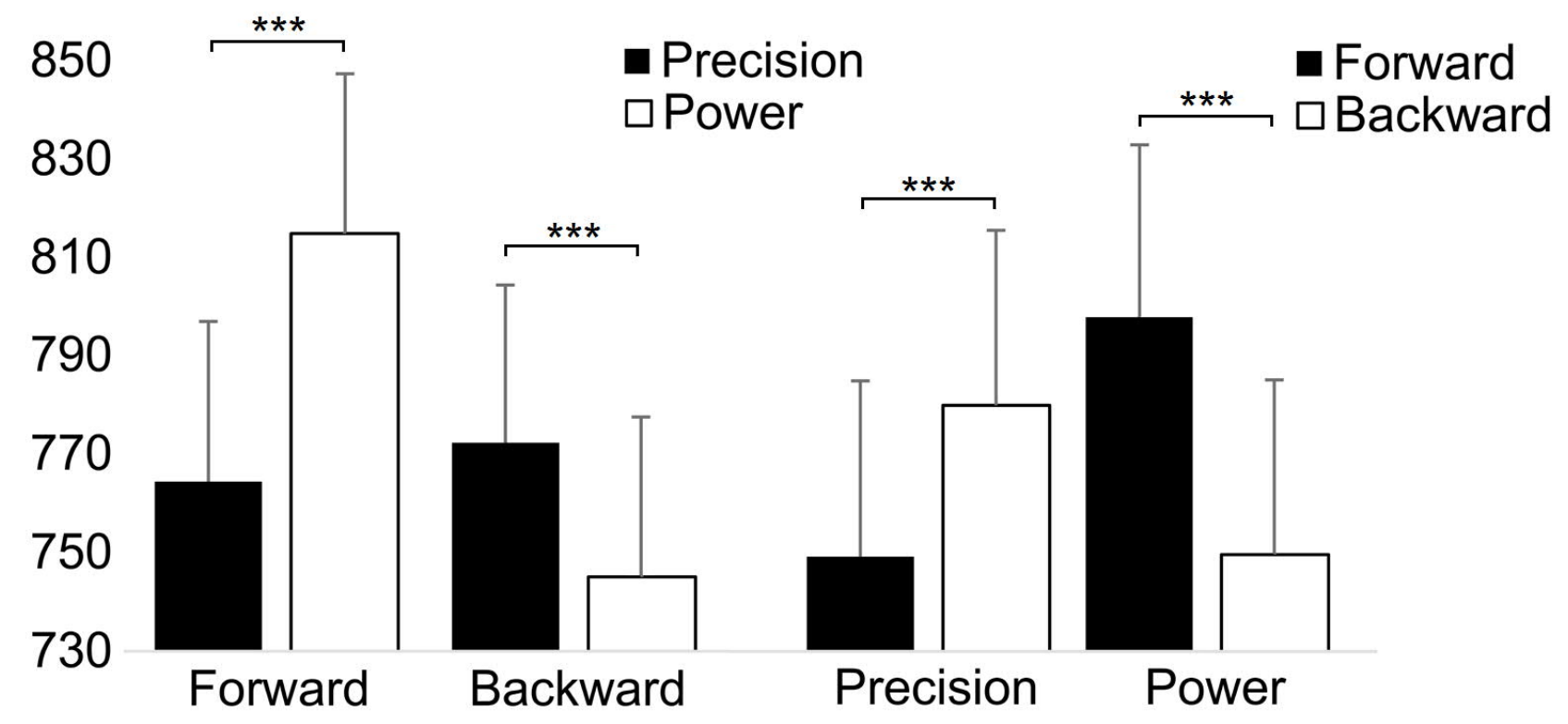

